\title{
Recurrent Novae: What Do We Know about Them?
}

\author{
G. C. Anupama \\ Indian Institute of Astrophysics, II Block Koramangala, Bangalore 560034, India \\ email: gca@iiap.res.in
}

\begin{abstract}
Recurrent novae (RNe) belong to the group of cataclysmic variables that exhibit nova outbursts at intervals on the order of decades. They are rare, with 10 Galactic RNe known to date. Two are known in the LMC, while there are a few suspected RNe in M31. Nova outburst models require a high accretion rate on a massive white dwarf to explain the recurring nova outbursts, making this class of objects one of the most likely progenitor binary systems of Type Ia supernovae. The observational properties of the known Galactic recurrent novae are presented here, together with some discussion on the recent outbursts of RS Ophiuchi (2006), U Scorpii (2010), and T Pyxidis (2011).
\end{abstract}

Keywords. stars: novae, cataclysmic variables, individual (RS Oph, T CrB, V3890 Sgr, V745 Sco, U Sco, V394 CrA, V2487 Oph, T Pyx, CI Aql, IM Nor)

\section{Introduction}

Recurrent novae (RNe) form a small group of cataclysmic binary systems that have recorded nova outbursts on recurrence timescales of a decade to a century, with an outburst luminosity $M_{V} \leqslant-5.5$. The binary components are a hot, massive white dwarf $\left(\mathrm{M}_{\mathrm{WD}} \geqslant 1.2 \mathrm{M}_{\odot}\right)$ accreting material from an evolved companion, with mass transfer rates $\sim 10^{-8}-10^{-7} \mathrm{M}_{\odot} \mathrm{yr}^{-1}$. The outburst is powered by a thermonuclear runaway in the accretion layer (e.g., Starrfield et al. 1985, Kato 2002, Yaron et al. 2005). There are ten known Galactic RNe, and a few more suspected ones, while two are known in the Large Magellanic Cloud and a few suspected systems in M31. Recent reviews on the properties of RNe can be found in Anupama (2008) and Schaefer (2010a). The multiple nova explosions make these systems ideally suited for the study and understanding of accretion processes and explosions in accreting systems. The presence of a massive, accreting white dwarf in these systems, with the possibility of the white dwarf increasing its mass, make them potential progenitors of Type Ia supernovae.

The basic parameters of the known Galactic RNe (Schaefer 2010a, Anupama 2008 and references therein) are listed in Table 1 . These parameters clearly indicate the heterogeneity in this small group, both during outburst and at quiescence. The speed class ranges from the very fast to the slow systems. The orbital periods range from a few hours, similar to classical nova systems, to a few hundred days as in the case of symbiotic binary systems, indicating the diversity in the nature of the mass losing secondary. In the following sections, the properties of the Galactic RNe will be discussed.

\section{Long Period Binaries}

The $\mathrm{T}$ CrB class of $\mathrm{RNe}$ are systems that have an $\mathrm{M}$ giant secondary, with orbital periods $\sim$ several hundred days. At quiescence, these systems are very similar to the 
Table 1. Basic parameters of galactic recurrent novae

\begin{tabular}{|c|c|c|c|c|c|c|c|c|}
\hline Name & $m_{\max }$ & $m_{\mathrm{min}}$ & $\begin{array}{l}t_{3} \\
{[\mathrm{~d}]}\end{array}$ & $\begin{array}{l}<t_{\mathrm{rec}} \\
{[\mathrm{yr}]}\end{array}$ & $\begin{array}{l}\text { distance } \\
{[\mathrm{kpc}]}\end{array}$ & secondary & $\begin{array}{l}P_{\text {orb }} \\
{[\mathrm{d}]}\end{array}$ & $\begin{array}{l}\text { Outburst } \\
\text { [year] }\end{array}$ \\
\hline \multicolumn{9}{|c|}{ Long Period Binaries - T Crb/RS Oph Class } \\
\hline $\mathrm{T} \mathrm{CrB}$ & 2.5 & 9.8 & 6 & 80 & 0.9 & M3 III & 227.67 & 1866,1946 \\
\hline $\mathrm{RSOph}$ & 4.8 & 11.0 & 14 & 22 & 1.6 & M0/2 III & 455.72 & $\begin{array}{l}1898,1907,1933 \\
1945,1958,1967, \\
1985,2006\end{array}$ \\
\hline V3890 Sgr & 8.1 & 15.5 & 17.0 & 27 & 7.0 & M5 III & 519.7 & 1962,1990 \\
\hline V745 Sco & 9.4 & 18.6 & 14.9 & 52 & 7.8 & M6 III & 510 & 1937,1989 \\
\hline \multicolumn{9}{|c|}{ Short Period Binaries - U Sco Class } \\
\hline $\mathrm{U}$ Sco & 7.5 & 17.6 & 4.3 & 22 & 12.0 & $\mathrm{~K} 2 \mathrm{IV}$ & 1.23 & $\begin{array}{l}1863,1906,1917 \\
1936,1979,1987, \\
1999,2010\end{array}$ \\
\hline V394 CrA & 7.2 & 18.4 & 5.5 & 38 & 10.0 & K & 1.52 & 1949,1987 \\
\hline $\mathrm{V} 2487 \mathrm{Oph}$ & 9.5 & 17.3 & 8 & $40 ?$ & 12.0 & & $\sim 1$ & 1900,1998 \\
\hline \multicolumn{9}{|c|}{ Short Period Binaries - T Pyx Class } \\
\hline $\mathrm{T} \mathrm{Pyx}_{\mathrm{x}}$ & 6.4 & $15.5^{\circ}$ & 62 & 19 & 5.0 & & 0.076 & $\begin{array}{l}1890,1902,1920, \\
1944,1966,2011\end{array}$ \\
\hline $\mathrm{CI} \mathrm{Aql}$ & 9.0 & 16.7 & 32 & 42 & 5.0 & K-M IV: & 0.6184 & $1917,1941,2000$ \\
\hline IM Nor & 8.5 & 18.3 & 80 & 82 & 3.4 & & 0.1026 & 1920,2002 \\
\hline
\end{tabular}

symbiotic binaries. The members of this group are fairly homogeneous in their outburst and quiescence properties.

The optical spectrum at quiescence is dominated by that of the giant secondary, with emission lines due to HI and HeI superposed. FeII, CaII and OI $8446 \AA$ lines are also present, except in T CrB. HeII lines are weak or absent (e.g. Anupama \& Mikolajewska 1999). The UV spectrum of $\mathrm{T}$ CrB shows a relatively hot continuum with emission lines and shell-like absorption features (Selvelli et al. 1992), while RS Oph shows a flat continuum with a few weak emission lines (Dobrzycka et al. 1996). Variability in the UBV/visual magnitudes, UV fluxes and optical emission line fluxes is detected on timescales of years (e.g. Iijima 1990, Anupama \& Mikolajewska 1999) and minutes (Zamanov et al. 2005). The long term variability is strongly correlated with the activity of the hot white dwarf that is embedded in an envelope of wind from the red giant (Anupama \& Mikolajewska 1999, Stanishev et al. 2004). The observed variability may be attributed to (a) fluctuations in the mass accretion rate and/or (b) changes in the column density of the absorbing optically thick wind envelope.

The outburst spectrum of this group is characterized by broad emission lines $\left(V_{\exp } \sim\right.$ $4,000 \mathrm{~km} \mathrm{~s}^{-1}$ ) that narrow with time (Figure 1). The early phase spectra show permitted lines, followed by the presence of intense coronal and other high excitation lines that develop when the nova has faded by 2-3 magnitudes from maximum, and fade as the nova enters the nebular phase. Hard and soft X-rays and radio emission, both thermal and non-thermal, have been observed in RS Oph during its recent outbursts in 1985 and 2006. The velocity deceleration, coronal lines and the non-thermal emission are well explained by the interaction of the nova material with the red giant wind material. The mass ejected during outburst is $\sim 3-4 \times 10^{6} \mathrm{M}_{\odot}$.

The 2006 outburst of RS Oph. RS Oph is the most well studied object amongst the long period systems, with the 2006 outburst being subject to mutiwavelength, multifacility observations (e.g. Bode et al. 2006, Sokoloski et al. 2006, Das et al. 2006, Evans et al. 2007a,b, Osborne et al. 2011, Eyres et al. 2009, Kantharia et al. 2007). All observations confirm the shock interaction of the expanding ejecta with the pre-outburst circumstellar material. X-ray, near-infrared and optical observations indicate the remnant was in the free expansion phase during the first $\sim 5$ days, and quickly moved to 


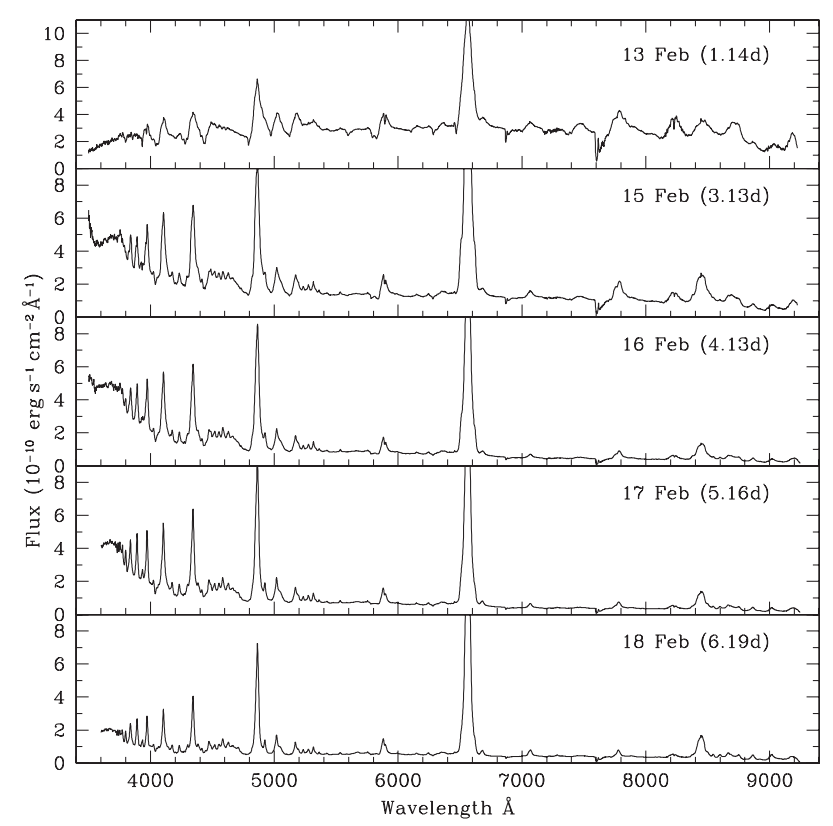

Figure 1. The early spectra of RS Oph during the 2006 outburst. Note the narrowing of the emission lines over the six days shown in the plot.

the radiative phase after a very brief adiabatic phase. The evolution of the non-thermal radio emission detected, for the first time, at low frequencies is very similar to supernovae (Kantharia et al.). The radio, X-ray and HST optical images (O'Brien et al. 2006, 2008, Bode et al. 2007, Ribeiro et al. 2009 and Luna et al. 2009) indicate the remnant to be bipolar. Radio images indicate a thermally dominated central region linked to expanding non-thermal lobes by a collimated outflow (Sokoloski et al. 2008). The optical images are modeled with two distinct co-aligned bipolar components, a low velocity, high density innermost region, with an extended high velocity structure (Ribeiro et al. 2009).

\section{Short Period Binaries}

\subsection{The U Sco Class}

The members of this class are very fast nova systems, with very similar outburst properties. U Sco is the most well studied member of this group, with its most recent, predicted outburst in 2010 (Schaefer 2010b) being extremely well monitored.

The 2010 outburst of U Sco. The outburst spectra are characterized by extremely broad emission lines (Figure 2) due to hydrogen Balmer, NIII, CIII and HeI lines with initial FWZI velocities of $\sim 10000 \mathrm{~km} \mathrm{~s}^{-1}$ (Yamanaka et al. 2010, Diaz et al. 2010, Kafka \& Williams 2010, Das et al. 2010, Anupama et al. 2011). The spectra indicate a rapid increase in the ionization levels, however, unlike in RS Oph, no coronal lines are seen in the U Sco systems. The nebular spectrum, observed for the first time (Diaz et al. 2010, Anupama et al. 2011) shows the presence of broad [OIII] and [NII] lines, and weak Balmer lines. U Sco exhibits a supersoft X-ray phase that begins around 9 days past maximum, and lasts until $\sim 40$ days (Schaefer 2010b). Coincident with the supersoft phase, a plateau is seen in the optical light curve. HeII lines emerge just before the onset of the supersoft phase (Anupama et al. 2011). The line profiles show a rapid evolution, with the broad 


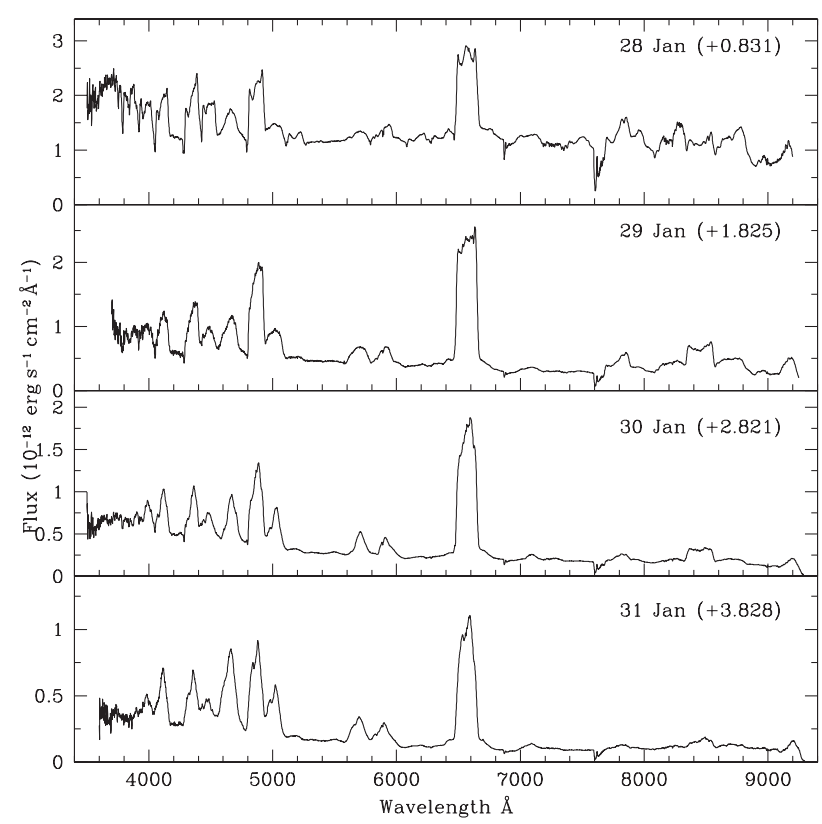

Figure 2. Optical spectrum of U Sco during the early phases of the 2010 outburst. Note the broad and boxy profile of the emission lines.

wings decreasing in strength with time. By $\sim 12$ days past maximum, the line profile is dominated by the sharp central component (Anupama et al. 2011). Flares of amplitude $0.1-0.5 \mathrm{mag}$ and duration of $\sim 1 \mathrm{hr}$ are seen during days $\sim 6-13$ (Worters et al. 2010, Schaefer 2010b). A spectrum obtained immediately after a flare shows narrow P Cygni absorption at $\sim 500 \mathrm{~km} \mathrm{~s}^{-1}$ in the HeI, CaII and N lines (Anupama et al. 2011).

Helium enrichment in the range $0.2-4$ has been estimated in the nova ejecta by Barlow et al. (1981), Williams et al. (1981), Sekiguchi et al. (1988), Anupama \& Dewangan (2000), while Iijima (2002) and Maxwell et al. (2011) find the helium abundance to be nearly solar.

Quiescence properties. The quiescence spectrum of the U Sco group is dominated by HeII lines (e.g. Hanes 1985, Duerbeck et al. 1993). Hydrogen lines are either absent or weak. U Sco has an evolved secondary, a K2 subgiant (Kahabka et al. 1999, Anupama \& Dewangan 2000, Schaefer 2010a). The orbital periods of V394 CrA and V2487 Oph indicate the presence of an evolved secondary in these systems also.

\subsection{The T Pyx Class}

T Pyx, CI Aql and IM Nor are all slow novae, with $t_{3}$ ranging from $36-80$ days. The outburst spectral development is however very similar in all three systems. Early spectra show lines due to HI, FeII, NIII and OI with P Cygni absorptions with velocities ranging from $\sim 800-2,500 \mathrm{~km} \mathrm{~s}^{-1}$. The spectral development is very similar to classical novae. Early spectra are similar to the FeII class, and changes to the $\mathrm{He} / \mathrm{N}$ class, similar to the hybrid novae. Coronal lines have been detected in CI Aql (Lynch et al. 2004) and T Pyx (Catchpole 1969). The mass ejected during outburst in these systems is very similar to the lower end of classical novae. CI Aql was detected as a weak, soft X-ray source, 14 and 16 months after the 2000 outburst (Greiner \& di Stefano 2002), while IM Nor was detected as a hard X-ray source 6 months after the 2002 outburst (Orio et al. 2005). 


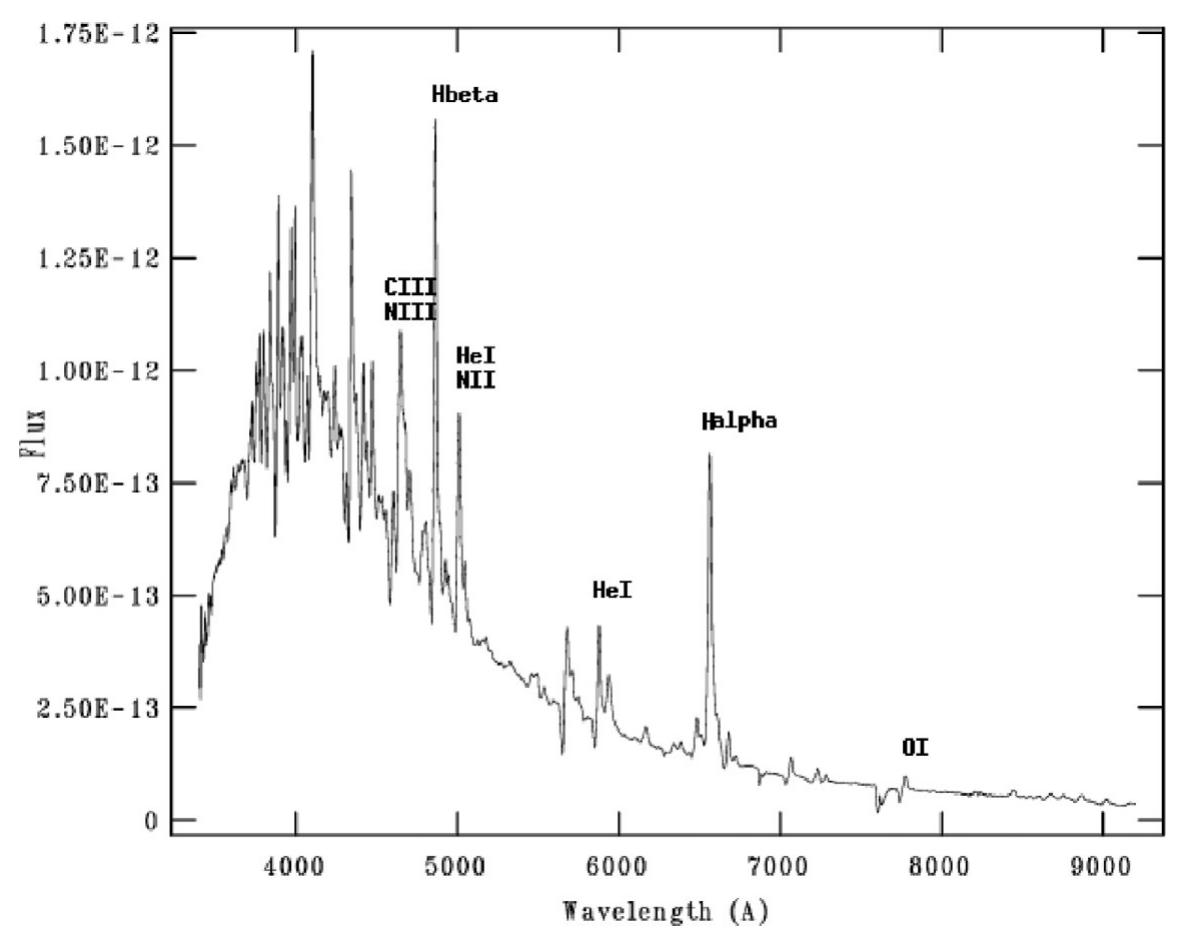

Figure 3. Spectrum of T Pyxidis on 15 April 2011.

The properties of T Pyx, CI Aql and IM Nor are quite different at quiescence. T Pyx and IM Nor have orbital periods that are similar to classical novae (Mennickent \& Honneycutt 1995, Uthas et al. 2010). T Pyx is the only recurrent nova with a shell that has a slow expansion velocity with thousands of discrete knots (e.g. Duerbeck \& Seitter 1979, Shara et al. 1997). CI Aql is an eclipsing binary with a quiescence spectrum that is very different from nova systems. The spectrum shows weak emission lines due to HeII, CIII-NIII complex on a reddened spectrum. Hydrogen Balmer lines are in absorption or absent (e.g. Anupama 2008), similar to shell stars. Spectra suggest a K-M secondary, while the orbital period suggest the secondary is evolved.

Although these systems are quite diverse in their quiescent properties, and probably in the nature of the binary components, they have been grouped together as a single class based on the similarities during outburst.

The 2011 outburst of T Pyx. T Pyx was discovered to have undergone its sixth outburst recently on 2011 April 14.2931 (Waagan 2011), on its rise to maximum. The early, low resolution optical spectrum obtained one day after discovery (Figure 3) shows emission lines due to hydrogen Balmer, NII, NIII, CIII, OI, HeI and CaII with P Cygni absorption at $\sim 1,400 \mathrm{~km} \mathrm{~s}^{-1}$. The high resolution spectra obtained on the same day (Shore et al. 2011) indicate the presence of He II line also. About a week later, Fe II lines developed, with discrete high negative radial velocity absorption components (Shore et al. 2011). X-ray emission has been detected (Kuulkers et al. 2011). The outburst is being closely monitored in all wavelengths with various facilities and one can expect several interesting results from these observations. 


\section{Light Curves of Recurrent Novae}

Comprehensive photometric histories of the Galactic RNe was recently presented by Schaefer (2010a). Based on detailed analyses of all available photometry of the RNe at quiescence as well as outburst, this work provides complete light curves and light curve templates for all eruptions and orbital periods. An extensive search for missed periods and an estimate of the recurrence timescales are also provided, which are reflected in Table 1.

Outburst light curves. The U Sco and T CrB systems are fast novae, with $t_{3} \leqslant 15$ days, while T Pyx, CI Aql and IM Nor are slow novae, with $t_{3} \geqslant 30$ days. Despite the differences in the decline rates, there are quite a few similarities in the properties of the outburst light curves of all RNe.

The light curves are same from eruption to eruption. This indicates that the eruption light curve depends on system parameters such as the white dwarf mass and composition that do not vary from eruption to eruption. All RNe, with the exception of $\mathrm{T} \mathrm{CrB}$, show a plateau in the light curve during decline. $\mathrm{T} \mathrm{CrB}$ has a secondary maximum. Other than the overall timescales, the declines from peak are similar for all systems. The early light curve indicates a power-law decline with an index of $\sim-1.75$, while the decline at the late phases has an index of $\sim-3.0$.

Quiescence light curves. The light curves of $\mathrm{RNe}$ at quiescence have a few typical, common characteristics. All RNe display flickering on time scales from minutes to hours, with amplitudes up to half a magnitude, a clear indication of accretion activity onto a white dwarf. The light curves also display large amplitude variations on time scales of months, years, decades, century. Except in the case of $\mathrm{T} \mathrm{CrB}$, no pre-eruption rise/dip is detected in the light curves.

CI Aql, U Sco, V394 CrA, V745 Sco and V3890 Sgr all have an identical pattern in their light curve folded about the orbital period. The maximum following the primary (deeper) minimum is significantly higher than the maximum following the shallower secondary minimum. The systems with giant secondaries (T CrB, V3890 Sgr, V745 Sco) show ellipsoidal variations.

The luminosities of RNe at quiescence range from $-4.1<M_{V}<3.2$ mag. Except the $\mathrm{T}$ CrB class, i.e. systems with giant secondaries, the quiescence luminosity is dominated by the accretion disk. The median luminosity at $2.2 \mathrm{mag}$ is brighter than the classical novae, implying a higher rate of accretion in RNe.

\section{The White Dwarf in Recurrent Novae}

Theoretical models suggest that the white dwarf in all RNe is hot and massive, accreting at a fairly high rate. Theoretical models for the optical and X-ray light curves (e.g. Hachisu \& Kato 2001, Hachisu et al. 2003) during outbursts indicate white dwarfs with mass near the Chandrasekhar limit for the T CrB and U Sco systems, and $\sim 1.2 \mathrm{M}_{\odot}$ for the T Pyx class short binaries. The observational estimates for the white dwarf in U Sco is $1.55 \mathrm{M}_{\odot}$ (Throughgood et al. 2000), $1.02 \mathrm{M}_{\odot}$ in CI Aql (Sahman \& Dhillon, these proceedings) and $>0.7 \mathrm{M}_{\odot}$ in $\mathrm{T}$ Pyx (Uthas et al. 2010).

The estimates of mass ejected during the outburst in the fast RNe systems, and theoretical models indicate that not all the accreted material is ejected during an outburst (e.g. Kahabka et al. 1999, Anupama \& Dewangan 2000, Hachisu et al. 2007). This implies that the white dwarf in these systems could increase its mass, and in the event of it being a $\mathrm{CO}$ white dwarf the increase in mass could lead to a supernova explosion. 


\section{Summary}

The Galactic recurrent novae form a small, heterogeneous class of nova systems with ten known members. The speed classes of RNe range from the slow to the very fast.

RNe can be grouped into (a) long period systems and (b) short period systems. The long period binaries are very homogeneous, with almost similar outburst and quiescence properties. The secondary in these systems is a red giant, with the hot accreting white dwarf primary embedded in the red giant wind. The short period binaries are a heterogeneous group, and be further sub-grouped. U Sco, V 394 CrA and V2487 Oph are very similar in their outburst and quiescence properties. These are very fast novae, with the ejecta possibly He enriched. The secondary in U Sco and V394 CrA is a subgiant. T Pyx and IM Nor are slow novae with similar properties. Both systems have an orbital period of the order of a few hours, similar to classical novae. CI Aql is a moderately fast nova with outburst properties very similar to T Pyx. It has been detected as an eclipsing binary, with a K-M evolved main sequence secondary, similar to U Sco. The quiescence spectrum is very peculiar, with a red continuum and shell star-like emission features.

All $\mathrm{RNe}$, with the exception of $\mathrm{T} \mathrm{CrB}$, show a plateau during the decline which is attributed to an accretion disc around the white dwarf which reappears a few to several days after optical maximum, irradiated by the white dwarf photosphere.

The white dwarf in RNe is more massive than commonly found in classical novae. The mass of the white dwarf is believed to be close to the Chandrasekhar limit in the fast systems, while it is lower $\sim 1.2 \mathrm{M}_{\odot}$ in the slow systems. The white dwarf mass in the fast systems is probably growing, making them likely supernova progenitors.

\section{References}

Anupama, G. C. 2008, in: A. Evans, M. F. Bode, T. J. O'Brien \& M. J. Darnley (eds.), RS Ophiuchi (2006) and the Recurrent Nova Phenomenon ASP Conf. Ser 401, (San Francisco: ASP), p. 31

Anupama, G. C. \& Dewangan, G. C. 2000, AJ, 119, 1359

Anupama, G. C. \& Mikołajewska, J. 1999, A\& $A$, 344, 177

Anupama, G. C. et al. 2011, MNRAS, (submitted)

Barlow M. J. et al. 1981, MNRAS, 195, 61

Bode, M. F. et al. 2006, ApJ, 652, 629

Bode, M. F. et al. 2007, ApJ (Letters) 665, L63

Catchpole, R. M. 1969, MNRAS, 142, 119

Das, R. K., Banerjee, D. P. K., \& Ashok, N. M. 2006, ApJ (Letters) 604, L129

Diaz M. P., Williams R. E., Luna G. J., Moraes M., \& Takeda L. 2010, AJ, 140, 1860

Dobrzycka, D., Kenyon, S. J., Proga, D., Mikołajewska, J., \& Wade, R. A. 1996, AJ, 111, 2090

Duerbeck, H. W., Duemmlerm R., Seitter, W. C., Leibowitz, E. M., \& Shara, M. M. 1993, Ann. Israeli Phys. Soc., 10, 284

Evans, A., et al. 2007a, MNRAS, 374, L1

Evans, A., et al. 2007b, ApJ (Letters) 671, L157

Eyres, S. P. S., et al. 2009, MNRAS, 395, 1533

Greiner, J. \& Di Stefano, R. 2002, ApJ, 578, L59

Hachisu, I \& Kato, M. 2001, ApJ, 558, 323

Hachisu, I., Kato, M., \& Luna, G. J. M. 2007, ApJ (Letters) 659, L153

Hachisu, I., Kato, M., \& Schaefer, B. M. 2003, ApJ, 584, 1008

Hanes, D. A. 1985, MNRAS, 213, 443

Iijima, T. 1990, J. Am. Assoc. Variable Star Obs. 19, 28

Iijima, T. 2002, $A \mathscr{E} A, 387,1013$

Kafka S., Williams R. 2011, A\& A, 526, 83

Kahabka, P., Hartmann, H. W., Parmar, A. N., \& Neueruela, I. 1999, A\& A, 347, L43 
Kantharia, N. G., Anupama, G. C., Prabhu, T. P., Ramya, S., Bode, M. F., Eyres, S. P. S., \& O'Brien, T. J. 2007, ApJ (Letters), 667, L171

Kato, M. 2002, in B.T. Gänsicke, K. Beuermann \& K. Reinsch (eds.), The Physics of Cataclysmic Variables and Related Objects, ASP Conference Series 261, (San Francisco: ASP), p595

uuljers, E., et al., 2011, ATel, 3285, 1

Luna, G. J. M., Montez, R., Sokoloski, J. L., Mukai, K., \& Kastner, J. H. 2009, ApJ, 707, 1168

Lynch, D. K., et al. 2004, AJ, 127, 1089

Maxwell M. P., et al. 2011, MNRAS, (accepted)

Mennickent, R. E. \& Honeycutt, R. K. 1995, IBVS, No. 4232

O'Brien, T. J., et al. 2006, Nature, 442, 279

Orio, M., Tepedelenlioglu, E., Starrfield, S., Woodward, C. E., \& Della Valle, M. 2005, ApJ, 620,938

Osborne, J. P., et al. 2011, ApJ, 727, 124

Ribeiro, V. A. R. M., et al. 2009, ApJ, 703, 1955

Schaefer B. E. 2010, ApJS, 187, 275

Schaefer B. E. 2010b, astro-ph.SR, arXiv:1009.3197

Sekiguchi K., Feast M. W., Whitelock P. A., Overbeek M. D., Wargau W., Spencer Jones J. 1988, MNRAS, 234, 281

Selvelli, P. L., Cassatella, A., \& Gilmozzi, R. 1992, ApJ, 393, 289

Shara, M., et al. 1997, AJ, 114, 258

Shore, S. N., Augusteijn, T., Ederoclite, A., \& Uthas, H. 2011, A\&\&A, 533, L8

Sokoloski, J. L., Luna, G. J. M., Mukai, K., \& Kenyon, S. J. 2006, Nature, 442, 276

Sokoloski, J. L., Rupen, M. P., \& Mioduszewski, A. J. 2008, ApJ (Letters) 685, L137

Stanishev, V., Zamanov, R., Tomov, N., \& Marziani, P. 2004, A\&SA, 415, 609

Starrfield, S., Sparks, W. M., \& Truran, J. W. 1985, ApJ, 291, 136

Thoroughgood, T. D., Dhillon, V. S., Littlefair, S. P. Marsh, T. R., \& Smith, D. A. 2001, MNRAS, 327, 1323

Uthas, H., Knigge, C., \& Steeghs, D. 2010, MNRAS, 409, 237

Waagan, E. 2011, CBET, 2700,1

Williams, R. E., Sparks, W. M., Gallagher, J. S., Ney, E. P., Starrfield, S. G., \& Truran, J. W. 1981, ApJ, 251, 221

Worters H. L., Eyres S. P. S., Rushton M. T., Schaefer B. 2010, IAUC 9114, 1

Yamanaka M., et al. 2010, PASJ, 62, L37

Yaron, O., Prialnik, D., Shara, M. M., \& Kovetz, A. 2005, ApJ, 623, 398

Zamanov, R., Gomboc, A., Bode, M. F., Porter, J. M., \& Tomov, N. A. 2005, PASP, 117, 268 